\title{
GLAD!
}

Revue sur le langage, le genre, les sexualités

$06 \mid 2019$

Varia

\section{La Pérille Mortelle}

Mortal Dangeresse

\section{Typhaine D}

\section{(2) OpenEdition}

Journals

Édition électronique

URL : http://journals.openedition.org/glad/1489

DOI : $10.4000 /$ glad. 1489

ISSN : 2551-0819

\section{Éditeur}

Association GSL

\section{Référence électronique}

Typhaine D, «La Pérille Mortelle », GLAD! [En ligne], 06 | 2019, mis en ligne le 01 juillet 2019, consulté le 17 décembre 2020. URL : http://journals.openedition.org/glad/1489; DOI : https://doi.org/10.4000/ glad. 1489

Ce document a été généré automatiquement le 17 décembre 2020.

\section{(c) (i) (9)}

La revue GLAD! est mise à disposition selon les termes de la Licence Creative Commons Attribution Pas d'Utilisation Commerciale - Pas de Modification 4.0 International. 


\title{
La Pérille Mortelle
}

\author{
Mortal Dangeresse
}

\section{Typhaine D}

\section{NOTE DE L'AUTEUR}

On peut retrouver la vidéo de cette intervention en ligne, sur la chaîne YouTube de Typhaine D, ainsi que d'autres informations sur ses actualités, spectacles, livres, etc, sur son site internet : www.typhaine-d.com . Typhaine D remercie chaleureusemente les relectrices formidables de ce texte pour la présente édition : Géraldine Franck, Juliette Mercier, Claire Fougerol, JwhY, ainsi que toutes les personnes permettante que ce texte soit publié.

Typhaine D est autrice ${ }^{1}$, comédienne, metteuse en scène, coach, conférencière, professoresse de théâtre, et militante féministe.

Elle travaille sur la démasculinisation du langage, et la création d'une langue féministe, depuise des années. Elle a notammente inventée la concepte de « La Féminine Universelle » lors de l'écriture de son spectacle et livre Contes à Rebours. Cette réinvention féministe des contes de fées commence donc toute naturellemente par « Elle était une fois ».

« La Féminine Universelle » consiste en l'invention d'une grammaire féministe imPertinente, de mots inventés comme « Femmage » (qui a sûremente due exister par la passée), ou encore « Noues » et « Voues » pour rassembler la Peuple des Femmes, les « Sœurcières » et les « anniversœurs » pour fêter notre Sororité ; et toute autre liberté féministante qui semblera signifiante ou émancipatrice à l'autrice, ou simplemente qui lui plaira. En effête, les instigateurs de la masculinisation du langage sous Richelieu ne s'étante pas encombrés de logique ou d'objectivité dans leure sinistre tâche, nulle n'y est à son tour tenue dans la riposte. Par ailleures, la « La Féminine Universelle », débridée, insurgée, insubordonnée, se trouve en constante évolution, et n'est pas restreinte par des règles strictes, si ce n'est celle de toujoures être porteuse de sens allante vers les 
intérêts des Femmes, donc en lutte féministe radicale intransigeante contre toutes formes des violences ou d'arnaques masculinistes et misogynes. Chacune est donc chaleureusemente invitée à utiliser la « La Féminine Universelle » en tant qu'acte de résistance littéraire, artistique et militante, et d'y ajouter ses propres trouvailles, comme cela se fait d'ailleures déjà, notammente sur les réseaux sociaux. L'autrice en est toujoures très heureuse, et Voues remercie alors de « rendre à Cléopâtre ce qui est à Cléopâtre » en citant sa travaille. Car affirmer la maternité de nos œuvres à chacune consolide notre Matrimoine commune, et participe de nos Luttes féministes et sorores, contre l'effacement et la mise sous anonymat qui menace toujoures les créatrices, ainsi que contre l'appropriation et l'instrumentalisation de nos inventions de Femmes, de Féministes, par des hommes, et à nos dépens.

Typhaine D a écrite et interprétée pour la première fois ce texte « La Pérille ${ }^{2}$ Mortelle » à l'occasion du concours d'éloquence du Collectif Droits Humains pour Tou'te's, la 10 décembre 2017 à l'Hôtel de Ville de la Mairie de Paris. Le titre féminisé de ce texte intervient en résistance aux élucubrations affolées de l'académie française, qui déclarait en 2017, avec toute la mesure que nous connaissons aux « Immortels », que l'écriture inclusive constituait pour la langue française un « péril mortel » (rien que ça); ces blancs messieurs combattante la démasculinisation du langage en agitante fébrilemente les arguments les plus iniques qui soient. Une riposte s'imposait, taraudait l'autrice : la voilà ! Ce texte va plus loin que les précédents écrits de Typhaine D dans l'utilisation de « La Féminine Universelle ». Et de faite, c'est là toute son objective, d'aller, non pas la plus loin possible dans le souci de rester lisible, et parce que la démarche est par définition révolutionnaire et en permanente construction, maise d'aller loin, pour faire entendre profondémente ce masculin qui Noues étouffe au quotidien sous des semblantes d'évidence, insinué dans les mots et les règles qui Noues ont étée données pour dire, et donc pour penser. « La Pérille Mortelle » est une essaie, une manifeste de langage, en révolte, en chemin.

1 Messieurs, Mesdamoiseaux, Mesdames! Mes chères commatriotes! Oyez Oyez ce "Conte à Rebours"! Très à rebours même, un conte... à régler! Ça va donc saigner ! (Régler, saigner : évocation menstruelle, comme on dite, "de la meilleure goûtte"! Voilà qui apporte un peu de couleur, de matière, de musc, à une introduction digne de ce nom. Je Voues la conseille, en conférence, ça faite toujoures sa petite effête !). Breffe. Elle était une fois, dans un royaume très très... non maise tellemente ${ }^{3}$ lointain quoi, nommé le Matriarcat - d'aucune l'appelle même : la Matriarcate ! - une cardinale, à la robe rouge. La couleur si rouge de sa robe était certainemente due à l'exhibition, aux quatre points cardinaux justemente, de ses abondantes, majestueuses, excellentes, éminentes menstruations, et c'est ainsi qu'on l'appelait: son Éminence. Elle portait matronyme de RicheLieu-à-soie. Elle avait créée une rassemblemente de ses copines, qu'elle avait appelée l'Académie Française, et qui des siècles après allait encore casser les bonbons des hommes. Dans ce groupe de copines académiciennes, désormaise autonommées, avec cette humilité qui devait les caractériser toujoures, et ce malgré les preuves récurrentes de la contraire, les Immortelles, était une fois une grammairienne. Noues la nommerons Claude Favre de Vaugelasse. Celle-ci trouvait que la langue que l'on parlait alors dans ce pays n'était pas encore assez sexiste. Sachante que le langage est éminemmente politique et structure la pensée, elle avait énoncée la règle de « la 
féminine l'emporte sur la masculine ", au prétexte que : la genre féminine est la genre la pluse noble. Argumente irréfutable, s'elle en est, Voues en conviendrez.

2 Ainsi, fie de la règle de proximité ! Désormaise, pour bien parler Française, l'on se devait de dire : « Les Femmes et les Hommes sont Belles. »

3 Ainsi, fie de la règle de majorité ! L'on était tenue de dire : La Reine Louise XIV et son harem de dix mille courtisans sont assises.

4 Ainsi, la genre Féminine, genre des Femmes, fut égalemente décrétée genre neutre. L'on pourrait croire comme cela de prime aborde, que cette dernière décision n'eut que peu d'incidence. Bien à la contraire! Suite à cette règle, l'on imposa que les adverbes, participes passées avec l'auxiliaire avoir, et autres participes présentes, deviendraient invariables, neutres. C'est à dire qu'un homme parlante à un autre homme était tenu de dire par exemple :

5 «Heureusemente, j'ai comprise la sermonne de la prêtresse à la messe dimanche dernière, elle est certaine qu'Adam est né de la côte d'Ève, etc. »

6 Ainsi, des mots furent redéfinis selon la bonne vouloire de ces dames, détentrices du bon sens. Par exemple, ambassadeur ne désigna pluse un homme ayante pour haute fonction de porter les intérêts de la Reine ou de la Présidente du Républic à l'étrangère, ce terme signifiante désormaise : le mari de l'Ambassadrice.

7 D'ailleures pourquoi un homme exercerait-il ces fonctions, je Voues la demande?

8 Ainsi, des mots furent interdits, jusqu'à ce qu'on finisse par les oublier. Car aujourd'hui, si l'on parle bien naturellemente des métiers, des titres de Professoresse, de Médecine et d'Autrice, elle ne vient pluse à l'esprite de personne de dire professeur, médecin, et auteur! C'est ridicule, et puise, entre Noues, c'est moche! Non maise c'est vraie! Écoutez plutôt: Professoresse, Autrice... Voilà qui est digne, et belle! Alors que: professeur, auteur... eur eur... Ces mots arrachent nos oreilles! Et puise ma déesse quel lourdeur... Qu'ont donc les militants masculinistes contre la beauté ! Contre l'élégance, la légèreté, la pureté de la Française! Mesdamoiseaux, messieurs reprenez-vous! Que Diablesse, un peu de Hauteur ! Maise des auteurs ça non, elle n'en est pas question!

9 On doit donc dire Monsieur La Mairesse de Paris Yann Hidalgo, Monsieur la Procureuse du Républic, etc. C'est bien pluse claire comme ça. D'ailleures, la faite de féminiser ces fonctions redore leure blason et valorise les personnes qui les exercent, femmes ou hommes! Alors que masculiniser ces mots les rapetissent - physiquemente déjà puisqu'ils sont pluse courts! - : c'est la raison pour laquelle beaucoup d'hommes tiennent à être appelés Monsieur l'Avocate - ou encore Maitresse plutôt que " maître ", qui convient mieuse à un usage en classe d'école primaire, ou dans le cadre de relations extra-conjugales... - égalemente Monsieur La Cheffe de service, Monsieur La Doctoresse, etc...

10 Aujourd'hui, des associations de masculinistes prostatiques, pour reprendre la notion bien connue selon laquelle la prostate serait un petit être se promenante dans le corps des hommes, leure faisante perdre leures moyens régulièremente et les rendante donc trop faibles pour exercer les hautes fonctions et les métiers de l'esprite - voire de la Sainte-Esprite, car imagine-t-on UN cardinal, ou UN pape, soyons sérieuses - ces associations de lutte pour les droits des hommes donc, revendiquent des mots à la masculine. Pourtante, ils existent déjà ! Personne ne leure dispute «boulanger » pour désigner le monsieur à la caisse de la boulangerie pendante que la boulangère est aux 
fourneaux! Personne ne songe non pluse à interdire les noms de métiers... mettons voilà : assistant paternel, ou homme de chambre, hôte d'accueil, etc !

11 De surcroîte, à la Québec notammente depuise quelques temps, des hommes autrices revendiquent une forme masculine de ce nom de métier, que Noues tolérons si ça peut leure faire plaisir : autris (prononcer "autrice »). Si, c'est masculine autris! (elle épèle) A U T R I S... sans e ! Commente ça ne s'entend pas à l'orale ? Bien sûre que si ! Car ce n'est pas exactemente le même son «sss", entre "autrice » et "autris ». La première est pleine avec la «e » féminine qui l'ancre, Autrice, avec force et détermination comme ça ; la seconde en revanche est laissée virevoltante, indécise, en suspens : autri... sssss..? Rien ne vous empêche de faire un effort de prononciation! Maise ce raisonnable compromis ne saurait leure suffire, à ces hommes déchainés de chez Noues, rassemblés en asso haineuses de mal-b... de mâle (le mâle de la femelle je voulais dire, si, c'est ça que je voulais dire.) Ces farfelus revendiquent par exemple le mot «écrivain», pour parler d'un homme écrivaine!

Maise d'aborde, la genre féminine est neutre, Noues l'avons déjà dite, alors le débat n'a pas riche-lieu d'être!

13 Et puise, après toutes, les hommes écrivaines sont une espèce si rare, sont de telles exceptions à la règle générale qui veut que seules les Femmes créent de grandes Cuvres, qu'elle n'est pas utile d'avoir un mot pour les désigner ! Et je n'invente rien! J'en prends pour preuve notre Matrimoine littéraire! Ce n'est pas une hasarde si l'on étudie à l'école Victorine Hugotte et non Jules Drouet! Si l'on étudie Judith Shakespeare et non son frère hypothétique William (celui imaginé par Virginio Shewolf.) Est-elle bien nécessaire de créer un sobriquet spécial pour désigner la misérable poignée d'hommes qui se font prétention de gribouiller et gaspiller de l'encre à leures heures perdues? L'on ne va toute de même pas inventer un mot juste pour les frères Bronté, si ? "John Ayre" et "Les Hautes de Hurlevente», ça ne suffit pas à faire un patrimoine!

14 Tenez, encore un mot ridicule ça "patrimoine»! Elle y a des associations - la collective $\mathrm{F}-\mathrm{H}$ par exemple - qui veulent des journées du patrimoine en plusse de celles de la Matrimoine, qui suffisent pourtante amplemente !

Quand on pense qu'elle y a encore aujourd'hui des pays dans la Monde où les hommes n'ont pas encore le droit de voter ou de conduire une voiture... (Elle sanglote, puise s'indigne.) Ces hommes privilégiés de chez Noues feraient quand même mieuse de s'occuper de ces vrais combats, au lieu de perdre leure temps, et surtoute le nôtre, hein les copines, avec des histoires de bonshommes sur les mots à employer! Ces damoiseaux devraient s'occuper des choses pluse graves qui se passent ailleures !

Tiens, mondamoiseau... encore un joli mot qu'ils veulent Noues interdire! Si si! Une asso, Osez Le Masculinisme, a même faite une campagne pour demander qu'elle n'y ait pluse que Madame et Monsieur dans les documents administratifs... Alors que c'est de la galanterie "mondamoiseau ", c'est élégante! Ça veut dire que les hommes sont encore frais! D'ailleures Catherin Deneuf, et qu'on ne vienne pas me dire que ce grand acteur est sexiste, donc Catherin Deneuf a toujoures voulue qu'on l'appelle Mondamoiseau, justemente pour marquer son indépendance, et Moie, ça, je trouve ça bien comme combat! Monsieur, ça fait vieux. Plein de jeunes hommes se vexent d'ailleures si on les appelle Monsieur alors qu'ils ne sont pas mariés! Comme Catherin Deneuf, ils se sentent alors... Importunés! Et bien que ce soit un droit inaliénable, rassurez-Voues, pour les femmes que d'importuner l'homme - droit d'importuner sans 
lequel, on la sait, aucune amoure, aucune relation de séduction, aucune bonheure, aucune fleure, aucune pâquerette, aucune rayonne de soleille printanière ne serait pluse possible - j'essaie au maximum de ne pas vexer les hommes qui n'ont pas encore de propriétaire... heu d'épouse !! D'épouse bien sûre! Oui, "Monsieur " égratigne l'orgueil du damoiseau, cette vanité exquise et sensible de l'homme disponible... heu jeune !!! Jeune !! Qu'est-ce que j'ai bouffé Moie ce matin... Véridique, je la sais d'expérience, parce que, bonne, sans vouloir m'en vanter, je suis un peu ce qu'on appelle... une « Femme à hommes »...

Ah j'en ai connue dans ma longue vie des poulets, pis des jolis hein... on s'amusait bien dans le temps mesdames... l'âge d'or... quand aucun prostatique ne venait Noues importuner en beuglante des \#MeToo avec une demande d'âge minimum de consentemente... Voues allez voir qu'un jour, ils iront jusqu'à Noues parler de désire des hommes...

Alors aujourd'hui dans toute cela, on ne sait pluse commente draguer! Avec la menace de la délation, sous le prétexte phallucieux de la harcèlemente avec le hashtag \#balancetatruie... Ou \#balancetonagresseuse comme disent les masculinistes doublés d'une fantaisie animaliste, ou je ne sais pluse quelle ineptie...

Commente vous dites, monsieur dans le fond? Du coup faudrait dire aussi «Mademoiselle»? Maise ça n'existe pas ça! Et c'est ridicule! Noues c'est Madame depuise la naissance, voilà, c'est comme ça!

Et puise de toute façon, pour revenir au mot écrivaine, elle n'est pas convenable qu'un homme fasse profession d'écrire : c'est qui qui va garder les enfantes que Madame a eue la gloire si admirable de porter et de mettre à la Monde auparavante? N'est-elle pas normale que lorsque les Femmes ont choisie d'enfanter, choix qui ne peut leure revenir qu'à elles seules naturellemente, alors qu'elles ont risquée ainsi leure vie, avec tante de courage et de dignité, dans le sang et les cris de guerrières, et toute cela, parfois, pour forte peu de choses lorsqu'elles ont le malheur d'accoucher de garçons ! - Quel gâchis ! - N'est-elle pas normale donc, qu'après avoir menée cette noble guerre de la naissance, dont les hommes sont et seraient incapables d'affronter les affres et les douleurs, qu'elles aient pleinemente méritée de reprendre libremente leures vies de Femmes, pendante que leures hommes, leures pères, leures précédents fils ou leures frères s'occupent de la progéniture ! Enfine, y pense-t-on sérieusemente....

21 Avante, jadis, je ne dis pas, elle y avait des inégalités. Maise depuise que Charline De Gaule leure a offerte le droit de vote en France en 1944, de quoi se plaignent-ils encore ! Elle est pourtante bien généreuse avec eux, la France, notre père Matrie !

Et puise dans écrivain, on entend quand même vachemente "vain »! Je Voues ferais dire... Et puis pas le grand cru, maise une vaine vanité... Non, décidemente, c'est à en perdre

latine. Commente ça dans écrivaine on entend vaine aussi du coup ? Heu... Non. Non non là c'est pas pareille! À la limite oui ! Avoir de la veine... Finalemente, on entend presque écri-artère! Enfine arrêtez avec cette mauvaise foi! Et puise écrivaine, c'est belle. On ne peut pas dire la contraire.

Je leure ferai en plusse remarquer, à ces bonhommes, que dans écrivaine, le mot écrivain est contenu. De même dans médecine, le mot médecin y est, et prend même beaucoup de place ! À une lettre près je Voues jure, ces éternels insatisfaits, ils viennent Noues casser les ovaires des Académiciennes! 
D'ailleures, depuise quelques décennies, elle y a même des hommes à l'Académie! Si, au moins $2 \%$ ! Peut-être même plusse ! Je ne sais pas, je n'ai pas les chiffres, maise je leure rends femmage, à ces hommes mes consœurs académiciennes !

Enfine toute ça, cette prostatie généralisée, comme disait Gertude Freude : ça vient d'un complexe clitoridien... On la sait. Parce que eux, leur attirail mou et frappé, ça manque cruellemente de tenue et de classe! Et puis, ils font pipi avec... Et de toutes façons les masculinistes, sont tous des vieux-fils dont aucune femme n'a voulu, et c'est ça qui les rend hargneux. Et puise les hommes Voues les connaissez hein, ils ont toujoures une cupidité face à la pouvoire... et à l'argente... c'est dégoutante.

De toutes façons, la Première Ministre Edouarda Philippine a tranchée ! - Tranchée... Oh! Ne vous effarouchez pas mesdamoiseaux, je veux dire, tranchée... le débat !

Alors oui, je les entends d'ici Noues dire que justemente, on a eue jusqu'ici que des Présidentes du Républic, et que justemente la Présidente Macronne leure avait promise UN Première Ministre et puise finalemente elle a juste prise la personne la pluse compétente et voilà ! Et c'est tombée sur une femme, maise c'est une hasarde, faut pas voir la femelle partoute!

Et si l'avise de Mesdames Macronne ou Philippine ne suffisait pas, Noues pouvons Noues reposer aussi sur la personne la pluse à même de Noues éclairer sur cette importante question du langage, à savoir Jeanne-Michèle Blanquère, la Ministre de l'éducation! Rappelez-Voues ses sages paroles à l'Assemblée Nationale la 15 novembre dernière, pour rappeler qu'elle n'est nul besoin de déféminiser la langue, puisqu'enfine, et je la cite : "La France a comme emblème un homme, Marius (celui coiffé de la calotte phrygienne Voues savez), et l'un de ses pluse beau mot est masculin : le Républic!»

Brillante n'est-ce pas ? Et je Voues rappelle qu'à l'Assemblée, à ces paroles de raison et de clairvoyance, toutes ont applaudite. Ah... Ça me fait du bien de penser que nos enfantes sont entre ses mains... je l'aime bien cette Ministre...

Malheureusemente, loin de se reposer sur la Blanquère, enfine je veux dire, sur ses avise éclairées, voilà que maintenante j'entends de-ci de-là que les masculinistes veulent dire "Droits de l'Humaine », ou "Droits des Personnes Humaines », ou même carrémente «Droits Humains ", au lieu de la formule historique légitime : « Droits de la Femme ! » Maise où va-t-on? On a beau leure répéter que c'est «Droits de la Femme » avec une grande F Majuscule, ils ne sont jamaise contents ces bonshommes je Voues dis...

31 Et pourquoi pas «droits de l'homme » tante qu'on y est! (Elle rit à cette incongruité.) Alors oui, historiquemente c'est vraie, quand Robespierrette, Dantonne, Maratte, et leures copines ont écrite la déclarationne sacrée des Droits de la Femme et de la Citoyenne, c'est vraie qu'à l'époque cela voulait vraimente dire que seules les femmes étaient concernées, les hommes étante considérés mineurs à vie, sous l'autorité de leures mères puise de leures épouses. En même temps, faut les comprendre les révolutionnaires: n'en déplaise au demi-mondain olyn de Gougeon, si les hommes avaient votée en ce temps là, ils auraient tous étée pour la Papesse et pour la Reine! Aucun libre arbitre ces bigots, ces crapeaux de bénitiers! Ils auraient votée comme la Prêtresse du village! Et alors on n'aurait pas aujourd'hui la belle démocratie qu'on connait hein ! Liberté, Égalité, Sororité !! 

néologisme superflu, c'est de la couillerie ! La fraternité n'existe pas. Et le mot Sororité suffit parfaitemente pour exprimer l'idée magnifique selon laquelle: Noues sommes Toutes des Sœurs! frontons des Mairies! Exactemente comme ceux sur la Panthéonne tiens: "Aux grandes Femmes la Matrie reconnaissante »! Panthéonne où elle y a déjà 5 hommes, alors hein, c'est bien assez, maise qu'est-ce qu'ils veulent encore ? Ces masculinazis !!! Alors pour la dernière fois, "Droits de la Femme" avec une grande F majuscule, aujourd'hui toute la Monde sait bien que les hommes ont étée comprises dedans! Ils sont là, si si, les zozoms, bien confortablemente installés, bien protégés avec leure petit $\mathrm{h}$ minuscule derrière cette grande $\mathrm{F}$ qui cache la forêt !

35 Je ne vois pas pourquoi ça changerait! Déjà, c'est une honneure pour vous mesdamoiseaux, messieurs, d'être élevés au rang de Femmes, en étante inclus dans la dite déclarationne!

Moie, je vais Voues dire, je suis une Femme. Et je ne suis pas misandre hein... maise !!!

\section{NOTES}

1. Autrice est un mot légitime et historique. Il Noues a étée tour à tour volé par Richelieu et ses sbires de l'Académie au 17ème siècle, puis rendu dans les années 2010 par l'autrice, chercheuse, metteuse en scène, comédienne Aurore Evain, dont la travaille passionnante est à découvrir ! De même, les ouvrages et interventions brillantes de la linguiste et historienne Eliane Viennot, dont son livre Non, le masculin ne l'emporte pas sur le féminin, ont été une formidable source d'inspiration, de connaissances et d'encouragementes pour Typhaine D, dans son processus de création de La Féminine Universelle.

2. Prononcer « Périle».

3. Les adverbes, participes présentes, et participes passées avec l'auxiliaire avoir, sont ici invariables, neutres - donc féminine, la féminine étante neutre, hein, bien sûre. De même, les pronoms comme «leure » s'accorderont en nombre seulemente (leures à la plurielle), maise se passeront de marquer la forme masculine, précision superflue. 
INDEX

Thèmes : Créations

Mots-clés : écriture inclusive, langue en danger, académie française

Keywords : inclusive writing, language in danger, French academy

\section{AUTEUR}

TYPHAINE D

Typhaine D est autrice, comédienne, metteuse en scène, coach, conférencière, professoresse de théâtre et militante féministe. 